\title{
Coagulopathy In Covid-19: A Case Series
}

\author{
Samia Kazmi ${ }^{1}$, Fivzia Herekar ${ }^{1}$, and Samreen Sarfaraz ${ }^{1}$ \\ ${ }^{1}$ The Indus Hospital
}

June 25, 2020

\begin{abstract}
Covid-19 with its associated complications of overt disseminated intravascular coagulation (DIC), acute respiratory distress syndrome (ARDS) and multi organ failure have collectively become the end point of disease course. This case series provides an insight on the fulminant disease course and the management challenges faced.
\end{abstract}

\section{Title: Coagulopathy In Covid-19: A Case Series.}

Samia Kazmi ${ }^{1}$, Fivzia Herekar ${ }^{2}$, Samreen Sarfaraz ${ }^{3}$

1 Department of Internal Medicine, The Indus Hospital, Karachi, Pakistan.

2 Department of Internal Medicine, The Indus Hospital, Karachi, Pakistan.

3 Department of Infectious Disease, The Indus Hospital, Karachi, Pakistan.

\section{Corresponding Author:}

Dr. Samia Kazmi,

Department of Internal Medicine,

The Indus Hospital, Plot C-76, Sector 31/5,

Opposite Darussalam Society, Korangi Crossing,

Karachi -75190, Pakistan.

E-mail: samia.kazmi@tih.org.pk

Mobile: 0092-306-1608016

Abstract Word Count: 241

Manuscript Word Count: 1388

Number of Tables: 1

Number of Figures: 2

Abstract: Covid-19 with its associated complications of overt disseminated intravascular coagulation (DIC), acute respiratory distress syndrome (ARDS) and multi organ failure have collectively become the end point of disease course in the background of vascular thrombosis and microangiopathy. Elevated D-dimer and Ferritin level with derangement in coagulation profile have become prognostic markers for Covid- 19 .

This series of four cases provides an insight on thrombotic complications despite adequate thrombo prophylaxis with resultant overt DIC diagnosed based on International Society on Thrombosis and Hemostasis 
(ISTH) scoring system. The medical records of four cases of Covid-19 patients with resultant thrombotic complications and DIC were reviewed retrospectively to record all clinical information. ISTH scoring system was applied for the diagnosis of overt DIC in all patients.

All four cases included in this series expired secondary to overt DIC and multi organ failure. Failure to prevent thrombosis with subsequent bleeding complications due to

derangment in coagulation profile was observed in all four. One of cases developed large vessel stroke and pulmonary thrombi despite anti coagulation. Arterial thrombus was diagnosed in one case with resultant bleeding complications. Overt DIC remained the fulminant end-point of the disease course.

The purpose of reporting this case series is to highlight the thrombotic complications manifested during the disease course despite adequate anti coagulation warranting the need for alternative therapies. Overt DIC, occurring on median day five of admission, remained the end-point in all four cases posing a major challenge in disease management.

Keywords: Covid-19; SARS-CoV-2, Coagulopathy, Disseminated Intravascular Coagulation, Anticoagulation, Thrombosis

\section{Key Clinical Message:}

The purpose of reporting this case series is to highlight the thrombotic complications manifested during the disease course despite adequate anti coagulation warranting the need for alternative or escalation of established therapies.

\section{Introduction:}

Covid-19 has proven to be a multi-faceted disease in terms of its presentation and varied complications ranging from acute respiratory distress syndrome to disseminated intravascular coagulation setting new challenges for medical community worldwide. Coagulopathy in association with thrombotic complications and resultant disseminated intravascular coagulation (DIC) have become the hallmark of severe acute respiratory syndrome coronavirus 2 (SARS-CoV-2). Klok et al. evaluated a cohort of 184 ICU patients in which the reported cumulative incidence of thrombotic complications was $31 \%$, pulmonary emboli (PE) had the highest occurrence despite standard thromboprophylaxis[1] Related studies have also concluded high prevalence of thrombotic complications in Covid-19 patients intensifying the role of thromboprophylaxis as an indispensible part of management [2-6]

The ultimate outcome of coagulopathy disseminated intravascular coagulation (DIC), primarily its prothrombotic variant, has posed management challenges in Covid-19 patients. Several studies have predicted poor outcomes in association with markedly high levels of D-dimer, a fibrin degradation product (FDP) validating its prognostic role in disease management $[7,8]$

The current guidance with regard to prevention of thrombosis and management of coagulopathy and DIC recommends pharmacological thromboprophylaxis be given to all immobilized and severely ill patients with COVID-19 unless otherwise contraindicated[9]. For management of DIC, International Society on Thrombosis and Hemostasis (ISTH) score assessment should be made and management, guided according to clinical manifestation of DIC.

The following are two cases of thrombotic complications with superimposed DIC and two of overt DIC encountered in Covid-19 patients admitted in our facility despite adequate thromboprophylaxis.

\section{Case History:}

\section{Case 1:}

51 year-old male, physician, known case of hypertension and diabetes mellitus, was admitted with a 10 day history of cough and flu like symptoms. Vital signs on presentation were Temperature ( $\mathrm{T}) 37^{\mathrm{O}}$ Celsius, Heart rate (HR) 130, Blood Pressure (BP) 167/93, Respiratory rate (RR) 45, and Oxygen saturation $\left(\mathrm{SpO}_{2}\right)$ of 70 
$\%$ on room air. Clinical characteristics and laboratory parameters on presentation are mentioned in Table 1. PCR assay for Covid-19 was positive. Due to very high D-dimer levels $(>15,000 \mu \mathrm{g} / \mathrm{L})$ Low molecular weight heparin (LMWH) was initiated in prophylactic dose from Hospital day (HD) 1. Due to hypoxemic respiratory failure, he was ventilated on $\mathrm{HD} 2$ at which time his $\mathrm{PaO}_{2} / \mathrm{FiO}_{2}$ ratio was 65.5. On $\mathrm{HD} 10$, he developed new onset right sided hemiplegia and ptosis. CT head plain revealed acute ischemic infarct in the left middle cerebral artery territory i.e. involving the fronto-parietal region (Figure 1). Aspirin and high-dose statins were started. Computer tomography pulmonary angiogram (CTPA) done on the same day showed bilateral pulmonary artery thrombosis (Figure 2). However, due to the acute stroke, full dose of anticoagulation was withheld initially and started 48hours later after reevaluation with neuro imaging to rule out extension or bleed within the infarct. Venous Doppler ultrasonography of lower extremities excluded venous thromboembolism and Echocardiography was normal with no arrhythmias noted on ECG. Subsequently, he was taken on heparin infusion, with strict activated partial thromboplastin time (aPTT) monitoring, which was with held after 12 hours as he developed melena. He was resuscitated with blood products including fresh frozen plasma (FFP). He continued to exhibit clinical worsening with derangements in coagulation profile by HD 14. Prothrombin time / International Normalized ratio (PT/INR) was $26.1 \mathrm{sec} / 2.49$ and a diagnosis of overt-DIC based on ISTH scoring system was made. He continued to deteriorate eventually developing multi-organ failure requiring single session of hemodialysis and superimposed candidemia leading to death by sixteenth day of admission.

\section{Case 2:}

72 year-old male, ex-smoker with no prior co morbidities was admitted with a 4 day history of shortness of breath. His vital signs on presentation were T $37^{\circ}$ Celsius, HR 123, BP 144/64, RR 25, and $\mathrm{SpO}_{2}$ of $82 \%$. Chest X-ray showed bilateral infiltrates in the lower zones with PCR assay for Covid-19 reported positive. Standard thromboprophylaxis was initiated. Due to worsening oxygenation, he was electively intubated and ventilated on $\mathrm{HD} 2$ at which time his $\mathrm{PaO}_{2} / \mathrm{FiO}_{2}$ ratio was 80. On $\mathrm{HD} 3$, he was taken on vasopressor support as a presumed diagnosis of septic shock was made. On HD 4, his platelet count dropped to $30,000 / \mathrm{mm}^{3}$ but no active bleeding was observed; LMWH was with held. On HD 7, he developed bleeding from endotracheal tube and was transfused with blood products including platelets. On the same day, he developed progressive left thumb cyanosis and impending gangrene at the side where his arterial line was placed. Ultrasound color Doppler revealed thrombus in left radial and ulnar artery and heparin infusion was initiated. He expired on HD 10 due to multi-organ failure including acute renal shut down, refractory hypotension and overt DIC.

\section{Cases 3 and 4:}

38 and 65year male patients were admitted with a diagnosis of Covid-19 pneumonia (cases 3 and 4 in Table 1) Chest x-ray of both patients showed ground glass infiltrates with predilection to lower lung fields. For both cases, invasive ventilation was initiated due to high oxygen requirements and as D-dimer levels were high, thromboprophylaxis was commenced with unfractionated heparin (UFH) 5000 units Q12H as LMWH was unavailable at the time. On HD 9, case 3 developed derangements in coagulation profile with PT/INR at $41.4 \mathrm{sec} / 3.94$ and aPTT of $>2$ minutes, bleeding complications developed and FFP was transfused. Due to development of overt-DIC and septic shock, patient expired on day tenth of admission. In case 4, cardiac arrhythmias developed on HD 2 followed by high $\mathrm{FiO}_{2}$ requirements. On $\mathrm{HD} 3$, PT/INR was reported as 26.9 $\mathrm{sec} / 2.56$, due to high risk of bleeding UFH was with held. On the following day, patient went into cardiac arrest with mild bleeding from endotracheal tube, efforts to resuscitate him failed and he expired on HD 4.

\section{Discussion:}

In summary, cases 1 and 2 developed thrombotic complications despite standard thromboprophylaxis with resultant overt DIC seen in all four cases (median day 5 of admission) International Society on Thrombosis and Hemostasis (ISTH) score assessment was used for diagnosis of DIC and management guided accordingly, with overt DIC defined as more than or equal to 5 points. D-dimer, ferritin, C-reactive protein (CRP), platelet count, PT/INR, aPTT were routinely measured in all cases with the highest derangements in Ddimer levels followed by ferritin and PT/INR; notable derangements in coagulation profile found on median 
7.5 days. Case 4 exhibited the highest level of ferritin and CRP. Predicted fatal outcomes have been reported in occurrence with high levels of D-dimer and FDP further validating the use of these parameters as a risk stratification tool $[7,8]$

Neurological manifestations have also become a major part of Covid-19 entity, with many cases presenting as acute cereberovascular events, mostly involving large vessels and multiple territories[10-12]. Case 1 developed acute ischemic stroke on day tenth of admission initially managed with anti platelet therapy then later switched to heparin with evolvement into DIC as optimal anticoagulation targets could not be attained.

Anti coagulation has posed to be a double edged sword in Covid-19 making effective coagulation quite difficult to achieve. In preceding cases, bleeding complications developed due to failure of optimization of anti coagulation therapy. Despite adequate resuscitation, marked deterioration was observed in all cases leading to end point of multi-organ failure and DIC. Many studies have recommended higher dose of anticoagulation for critical Covid-19 patients[1, 4, 13] but as stated by American Society of Hematology (ASH), this remains controversial as there is no data supporting improved outcomes and some risks for VTE and bleeding are common. The Japanese Society of Thrombosis and Hemostasis have published a comprehensive consensus of management of DIC according to its type and has implicated the use of anti thrombin (AT) danaparoid sodium (DS) and synthetic protease inhibitor (SPI) in the asymptomatic-type DIC[14] Although measurement of AT levels was not undertaken at our facility but in a non randomized multi-institutional survey, the efficacy of AT concentrate in sepsis associated DIC was studied by Iba and colleagues which showed significantly improved rates of survival and recovery from DIC without an increased risk of bleeding[15] However clinical trials for the validation of such therapies in Covid-19 are still awaited.

At this point, desirable anticoagulation targets in Covid-19 remain idealistic to say the least, setting new challenges in the management of this disease. In the background of thrombotic complications and DIC, optimization of coagulation parameters remain a quixotic goal, warranting the need of escalation in anticoagulation therapy and consideration of experimental therapies as entailed above.

\section{Acknowledgements:}

There are no acknowledgements.

\section{Conflict Of Interest Statement:}

There are no conflict of interests in this study.

\section{Sources of Funding:}

None.

\section{Appendices:}

TABLE 1: Clinical characteristics and laboratory parameters of four patients at the time of admission

\section{VARIABLE}

Age-year

Sex

Medical history

Drugs given

Duration of Covid-19 symptoms-days

Outcome

White cell count $/ \mathrm{mm}^{3}$

Platelet count $/ \mathrm{mm}^{3}$

Pro thrombin time/INR-sec

Activated partial thromboplastin time-sec

CRP-mg/L

D-dimer- $\mu \mathrm{g} / \mathrm{L}$

\section{CASE 1}

51

Male

DM, HTN

HCQ, Methyl pred

10

Deceased

20.74

330,000

$11.3 / 1.08$

30.8

274

12,000 
TABLE 1: Clinical characteristics and laboratory parameters of four patients at the time of admission

\section{$\mathrm{LDH}-\mathrm{U} / \mathrm{L}$}

Ferritin-ng/mL

Worst laboratory parameter (value)

Thromboembolic event (occurred on HD)

ISTH score for DIC (calculated on HD)

D-dimer Ferritin $\mathrm{P}$ Left MCA thromb

$6(\mathrm{HD} 14)$

DM: Diabetes Mellitus, HTN; Hypertension, HCQ: Hydroxycholoroquine, IVIG: Intravenous Immunoglobulin, CRP: C-reactive protein, LDH: Lactate Dehydrogenase, DIC: Disseminated Intravascular Coagulation, ISTH: International Society on Thrombosis and Hemostasis scoring system for DIC includes platelet count, d-dimer level, pro-thrombin time in seconds and fibrinogen level ([?]5 points classifies as overt DIC), HD: Hospital day.

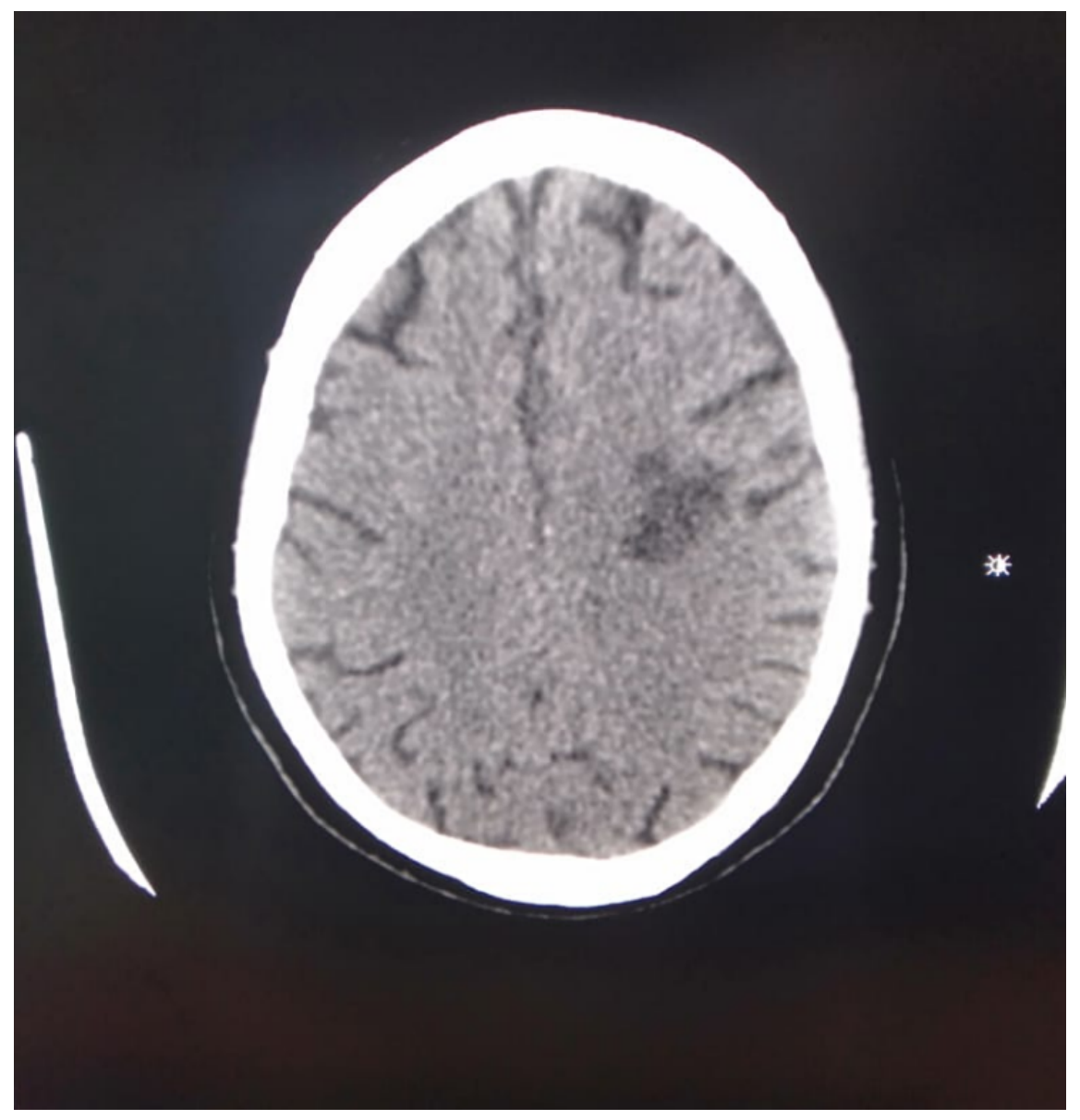

Figure 1: CT head plain film demonstrating acute ischemic infarct of the left middle cerebral artery territory. 


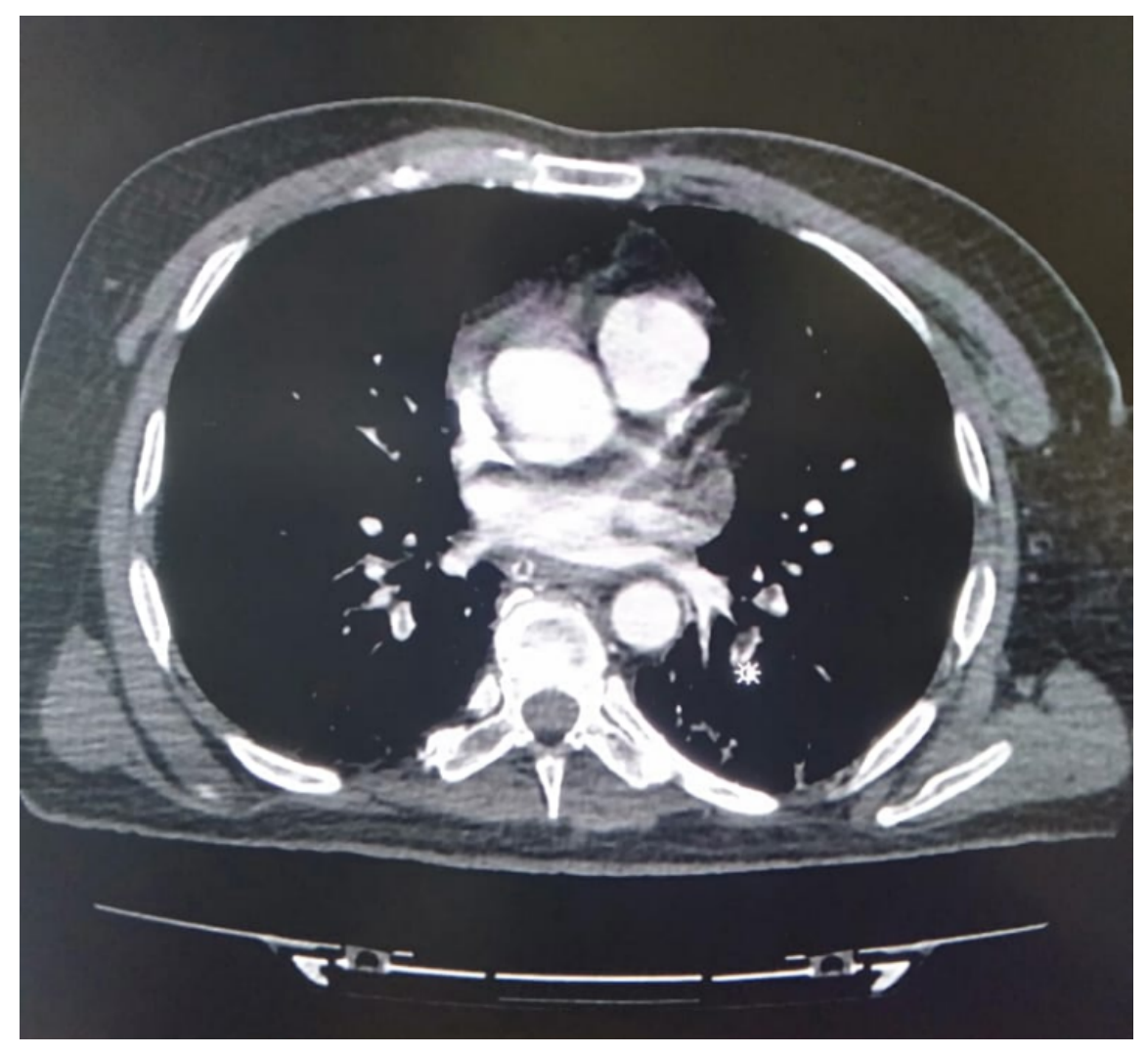

Figure 2: CT-pulmonary angiography (CTPA) demonstrating bilateral pulmonary artery thrombosis.

\section{References:}

1. Klok, F., et al., Incidence of thrombotic complications in critically ill ICU patients with COVID-19. Thrombosis Research, 2020.

2. Thomas, W., et al.,Thrombotic complications of patients admitted to intensive care with COVID-19 at a teaching hospital in the United Kingdom. Thrombosis Research, 2020.

3. Middeldorp, S., et al.,Incidence of Venous Thromboembolism in Hospitalized Patients with COVID-19. 2020 .

4. Helms, J., et al., High risk of thrombosis in patients in severe SARS-CoV-2 infection: a multicenter prospective cohort study. Intensive Care Med., 2020.

5. Cui, S., et al., Prevalence of venous thromboembolism in patients with severe novel coronavirus pneumonia. Journal of Thrombosis and Haemostasis, 2020.

6. Lodigiani, C., et al., Venous and arterial thromboembolic complications in COVID-19 patients admitted to an academic hospital in Milan, Italy. Thrombosis Research, 2020.

7. Zhou, F., et al., Clinical course and risk factors for mortality of adult inpatients with COVID-19 in Wuhan, China: a retrospective cohort study. The Lancet, 2020.395 (10229): p. 1054-1062.

8. Tang, N., et al., Abnormal coagulation parameters are associated with poor prognosis in patients with novel coronavirus pneumonia. Journal of Thrombosis and Haemostasis, 2020.18 (4): p. 844-847.

9. Thachil, J., et al., ISTH interim guidance on recognition and management of coagulopathy in COVID-19. Journal of Thrombosis and Haemostasis, 2020. 
10. Mao, L., et al., Neurologic Manifestations of Hospitalized Patients With Coronavirus Disease 2019 in Wuhan, China. JAMA Neurology, 2020.

11. Oxley, T.J., et al.,Large-vessel stroke as a presenting feature of Covid-19 in the young. New England Journal of Medicine, 2020: p. e60.

12. Beyrouti, R., et al.,Characteristics of ischaemic stroke associated with COVID-19.Journal of Neurology, Neurosurgery \& Psychiatry, 2020.

13. Llitjos, J.F., et al., High incidence of venous thromboembolic events in anticoagulated severe COVID-19 patients. Journal of Thrombosis and Haemostasis, 2020.

14. Wada, H., et al., Expert consensus for the treatment of disseminated intravascular coagulation in Japan. Thrombosis research, 2010. 125 (1): p. 6-11.

15. Iba, T., et al., Efficacy and bleeding risk of antithrombin supplementation in septic disseminated intravascular coagulation: a secondary survey. Critical Care, 2014.18 (5): p. 497. 